\title{
CONVERGENCE SETS IN REFLEXIVE BANACH SPACES
}

\author{
BRUCE CALVERT
}

ABSTRACT. A closed linear subspace $M$ of a reflexive Banach space $X$ with $X$ and $X^{*}$ strictly convex is the range of a linear contractive projection iff $J(M)$ is a linear subspace of $X^{*}$. Hence the convergence set of a net linear contractions is the range of a contractive projection if $X$ and $X^{*}$ are locally uniformly convex.

Let $X$ be a Banach space over $\mathbf{C}$ or $\mathbf{R}$, and let $\left(T_{n}\right)$ be a net of linear contractions on $X$. The convergence set for $\left(T_{n}\right)$ is $\left\{x \in X: T_{n} x \rightarrow x\right\}$. Bernau [1] showed that if $X$ is an $L_{p}$ space, $p \in(1, \infty)$, then a convergence set is the range of a linear contractive projection. A simplification and generalization of this result follows from the characterisation of ranges of contractive linear projections of Theorem 1, and is given as Theorem 2 below.

Let $S$ be a subset of $X$. Then the shadow of $S$ is the set of $x$ in $X$ such that $T_{n} x \rightarrow x$ for every net of linear contractions on $X$ such that $T_{n} y$ $\rightarrow y$ for all $y$ in $S$. Assuming $X$ to be an $L_{p}$ space, Bernau [1] showed that the shadow of $S$ is the range of a contractive projection, and that if $E$ is the range of a contractive projection, and $E$ contains $S$, then $E$ contains the shadow of $S$. This result holds generally, and is given as Corollary 2.

Theorem 3 considers finding the projection in terms of the net $\left(T_{n}\right)$.

By a nearest point projection (on a subset $K$ of a Banach space $X$ ) we mean a function $Q$ taking $x$ in $X$ to a nearest point in $K$.

Lemma 1. A set is the range of a linear contractive projection iff it is the nullspace of a linear nearest point projection.

Proof. $Q$ is a linear nearest point projection iff $I-Q$ is a linear contractive projection.

Theorem 1. Let $X$ be a strictly convex reflexive Banach space with strictly convex dual $X^{*}$. Let $J: X \rightarrow X^{*}$ be the duality map; $\|J x\|=\|x\|$, $(J x, x)=\|x\|^{2}$. Then a closed linear subspace $M$ of $X$ is the range of a

Received by the editors November 1, 1973.

AMS (MOS) subject classifications (1970). Primary 46B99, $47 \mathrm{~B} 99$.

Key words and phrases. Strictly convex, locally uniformly convex, contractive projection, convergence set, mean ergodic theorem. 
linear contractive projection iff $J(M)$ is a linear subspace of $X^{*}$.

Proof. Suppose $J(M)$ is linear. Let $Q$ be the nearest point projection on $J(M)^{\perp}$. (There exist nearest points since $X$ is reflexive, and only one since $X$ is strictly convex.) For $x \in X, Q x \in J(M)^{\perp}$ is defined by the property that for $y$ in $J(M)^{\perp},(J(x-Q x), y)=0$. (The real part of $J$ is the Gâteaux derivative of the function taking $x$ to $\|x\|^{2 / 2}$, since $X^{*}$ is strictly convex.) $J(M)$ is closed since $J^{-1}$ is continuous from the strong to the weak topology (since $X$ is reflexive and strictly convex). Hence, $Q x$ is defined by $Q x \in(J(M))^{\perp}$ and $J(x-Q x) \in J(M)$, or $x-Q x \in M$. This shows $Q$ is linear, for if $y \in X, y-Q y \in M, Q y \in(J(M))^{\perp}$, then $(x+y)-(Q x+Q y)$ $\in M$, and $Q x+Q y \in(J(M))^{\perp}$, giving $Q(x+y)=Q x+Q y$, and similarly $Q(\alpha x)$ $=a Q(x)$.

Since $Q x=0$ iff $x \in M$ the result follows from Lemma 1 .

Conversely, suppose $M=R(P)$, the range of a contractive linear projection. If $m \in M$,

$$
\left\|P^{*} J m\right\| \leq\|J m\| \leq\|m\|, \quad \text { and } \quad\left(P^{*} J m, m\right)=(J m, P m)=\|m\|^{2},
$$

giving $P^{*} J m=J m$, since $X^{*}$ is strictly convex. Hence, $J(M) \subset R\left(P^{*}\right)$. Replacing $P$ by $P^{*}$ and $J$ by $J^{-1}$ (since $X$ is strictly convex), $J^{-1} R\left(P^{*}\right) \subset$ $M$, giving $J(M)=R\left(P^{*}\right)$, completing the proof.

Corollary 0. Let $X$ be a reflexive Banach lattice with $X$ and $X^{*}$ strictly convex. Then a closed subspace $M$ is the range of a positive linear contractive projection iff $J M$ is a linear subspace and sublattice of $X^{*}$ iff $J M$ is a linear subspace and $M$ is a sublattice.

Proof. Let $P$ be a positive linear contractive projection. Since $P$ is positive, for $x$ in $X, P\left(x^{+}\right) \geq(P x)^{+}$. Replacing $x$ by $P x$ gives $P\left((P x)^{+}\right)$ $\geq(P x)^{+}$. Let $y$ be a convex combination of $P\left((P x)^{+}\right)$and $(P x)^{+}$. Since $X$ is a Banach lattice, $\|y\| \geq\left\|(P x)^{+}\right\|$. Since $\|P\|=1$, the opposite in equality holds. By strict convexity, $P\left((P x)^{+}\right)=(P x)^{+}$, which implies $M$ is a sublattice. The argument applied to $P^{*}$ gives $J(M)$ a sublattice.

Suppose $J M$ is a linear subspace and $M$ is a sublattice. For $x$ in $M$ and $y$ in $J(M)^{\perp}, x^{+} \in M$, giving

$\left\|x^{+}\right\|^{2}=\left(J\left(x^{+}\right), x\right)=\left(J\left(x^{+}\right), x+y\right) \leq\left(J\left(x^{+}\right),(x+y)^{+}\right) \leq\left\|x^{+}\right\|\left\|(x+y)^{+}\right\|$.

If $x+y \leq 0$, then $x \leq 0$. Since $X=M+J(M)^{\perp}$, the linear contractive projection on $M$ is positive. 
Corollary 1. Let $X$ be as in Theorem 1. Let $\left(M_{i}\right)_{i \in I}$ be a net of ranges of linear contractive projections. Then $M=\bigcup_{j} \bigcap_{i \geq j} M_{i}$ is the range of a linear contractive projection.

Proof. $J\left(\bigcup_{j}\left(\bigcap_{i \geq j} M_{i}\right)\right)=\bigcup_{j} \bigcap_{i \geq j} J\left(M_{i}\right)$ is a linear subspace since each $J\left(M_{i}\right)$ is.

Theorem 2. Let $X$ be a reflexive Banach space, with $X$ and $X^{*}$ locally uniformly convex. Then convergence sets are ranges of linear contractive projections.

Proof. Let $\left(T_{n}\right)$ be a net of contractions with convergence set $M$. If $m \in M,\left(T_{n}^{*} J m, m\right) \rightarrow\|m\|^{2}$, and $\left\|T_{n}^{*} J m\right\| \leq\|m\|$, giving $T_{n}^{*} J m \rightarrow J m$ since $X^{*}$ is locally uniformly convex. (If $Y$ is reflexive and locally uniformly convex, then given $y \neq 0$, and $\epsilon>0$, there exists $\delta>0$ such that if $\|x\| \leq$ $\|y\|$, and $\|x-y\| \geq \epsilon$, then $\|(x+y) / 2\| \leq\|y\|(1-\delta)$. Hence if a subsequence $T_{n^{\prime}}^{*} J m \rightarrow y$ weakly, then $\|y\|=\|m\|$. Since $\left\|T_{n^{\prime}}^{*} J m\right\| \rightarrow\|y\|,\left\|\left(T_{n^{\prime}}^{*} J m+y\right) / 2\right\|$ $\rightarrow\|y\|$, which implies $T_{n^{\prime}}^{*} J m \rightarrow y$. Since $y$ satisfies the inequalities defining $J m, y=J m$.) Hence, $M^{*} \supset J(M)$ where $M^{*}=\left\{f \in X^{*}: T_{n}^{*} f \rightarrow f\right\}$. Similarly, $M \supset J^{-1}\left(M^{*}\right)$, giving equality. The result follows by Theorem 1 .

Corollary 2. Let $X$ be as in Theorem 2. Let $S \subset X$. Then the shadow of $S$ is the smallest convergence set containing $S$.

Proof. By definition, the shadow of $S$ is the intersection of all convergence sets containing $S$, which is a convergence set by Theorem 2 and Corollary 1 .

Corollary 3. Let $X$ be as in Theorem 1 and $\left(T_{n}\right)$ a net of linear contractions. Then $\left\{x: T_{n} x \rightarrow x\right\}$, the weak convergence set, is the range of $a$ linear contractive projection.

Proof. By the proof of Theorem 2.

Lemma 2. Let $X$ be a reflexive Banach space, with $X$ and $X^{*}$ strictly convex. If $M$ is the range of a linear contractive projection $P$, it is the range of only one.

Proof. By Theorem $1, I-P$ is the nearest point projection on $N(P)=$ $R\left(P^{*}\right)^{\perp}=(J M)^{\perp}$.

Lemma 3. Let $X$ be a reflexive Banach space with $X$ and $X^{*}$ strictly convex. Given a finite set $\left(T_{n}\right)_{n \in F}$ of linear contractions, then the linear contractive projection on $\left\{x: T_{n} x=x\right.$ for $n$ in $\left.F\right\}$ is $\lim _{p \rightarrow \infty} \lim _{k \rightarrow \infty} A_{p, k}^{F}$ 
(in the strong operator topology), where

$$
A_{p, k}^{F}=\frac{1}{p} \sum_{j=0}^{p-1}\left(\prod_{n \in F} \frac{1}{k} \sum_{i=0}^{k-1} T_{n}^{i}\right)^{j},
$$

and the product can be taken in any order.

Proof. For $n$ in $F$, let $P_{n}$ be the linear contractive projection on $N\left(I-T_{n}\right)$, unique by Lemma 2. By the mean ergodic theorem, $T_{n}(k)=$ $(1 / k) \sum_{i=0}^{k-1} T_{n}^{i}$ converges to $P_{n}$.

By induction we show $\Pi_{n \in I} P_{n} x=x$, where $I \subseteq F$, implies $P_{n} x=x$ for $n$ in $I$. Suppose it is true for $m$ elements in $I$; then for $n$ in $F$, suppose $P_{n} \Pi_{i \in I} P_{i} x=x$. If $y$ is a convex linear combination of $x$ and $\prod_{i \in I} P_{i} x$, then $P_{n} y=x$, giving $\|y\|=\|x\|$. By strict convexity, $x=\prod_{i \in I} P_{i} x$, giving $P_{i} x=x$ for $x$ in $I$ by the inductive hypothesis, and hence $P_{n} x=x$. Hence,

$$
\bigcap_{n \in F} N\left(I-T_{n}\right)=\bigcap_{n} N\left(I-P_{n}\right)=N\left(I-\prod_{n \in F} P_{n}\right) .
$$

By the mean ergodic theorem, the nonexpansive projection on this set is $\lim _{p \rightarrow \infty}(1 / p) \sum_{j=0}^{p-1}\left(\Pi P_{n}\right)^{j}$. By continuity of multiplication of operators in the strong topology, we can take the limits outside, giving the formula.

Lemma 4. Let $X$ be a reflexive Banach space, and $A=\left(A_{n}\right)_{n \in S}$ a net of bounded linear operators on $X,\left\|A_{n}\right\| \leq M$ for all $n$. Define $N(A)=$ $\left\{x: A_{n} x \rightarrow 0\right\}$ and $R(A)=\left\{y:\right.$ there exists a subnet $\left(A_{n(m)}\right)_{m \in T}$ of $A$, a bounded set of $X,\left\{y_{n(m)}: m \in T\right\}$, and for $N$ in $T$ there is a set of positive numbers $\underset{m}{a_{m}^{N}}$ for finitely many $m \geq N$ in $T, \Sigma_{m} a_{m}^{N}=1$, and $y=$ $\left.\lim _{N} \Sigma_{m} \alpha_{m}^{N} A_{n(m)} y_{n(m)}\right\}$.

Then by defining $A^{*}=\left(A_{n}^{*}\right)$, we have $R(A)^{\perp}=N\left(A^{*}\right)$.

Proof. Take $f \in N\left(A^{*}\right), y \in R(A), y=\lim _{N} \Sigma_{m} a_{m}^{N} A_{n(m)} y_{n(m)}$, where $\left\|y_{n(m)}\right\| \leq K$ for all $m$. Then

$$
\begin{aligned}
(f, y) & =\lim \left(f, \sum_{m} \alpha_{m}^{N} A_{n(m)} y_{n(m)}\right)=\lim \left(A_{n(m)}^{*} f, \sum_{m} a_{m}^{N} y_{n(m)}\right) \\
& \leq \varlimsup \lim \left\|A_{n(m)}^{*} f\right\| K=0 .
\end{aligned}
$$

Suppose instead that $f \in R(A)^{\perp}$. We wish to show that if $T$ is a cofinal subset of $S$, then $\left(A_{n}^{*} \cap_{n \in T}\right.$. has a subnet converging to zero. Take $y_{n}=$ 
$J^{-1} A_{n}^{*} f$ for $n$ in $T$. By weak compactness, there is a weak cluster point $y$ for $\left(A_{n} y_{n}\right)_{n \in T^{*}}$ For $N$ in $T, y$ is in the weak closure of $\left\{A_{p} y_{p}: p \in T\right.$, $p \geq N\}$, and hence the strong closure of its convex hull. Thus for $U$ a neighborhood of $y$, we can take $\alpha_{p}^{N, U} \geq 0$, for $p \geq N, p$ in $T$, nonzero for only finitely many $p, \Sigma_{p} \alpha_{p}^{N, U}=1$, so that $\Sigma_{p} \alpha_{p}^{N, U} A_{p} y_{p} \in U$. Let $Q$ be the directed set of neighborhoods of $y$; then for $(p, U)$ in $T \times Q$, putting $A_{(p, U)}$ $=A_{p}$ gives $\left(A_{p, U}\right)_{(p, U) \in T \times Q}$ a subnet of $\left(A_{p}\right)_{p \in S}$ and

$$
y=\lim _{(N, U) \in T \times Q} \sum_{p} \alpha_{p}^{N, U} A_{p} y_{p},
$$

giving $y \in R(A)$. But

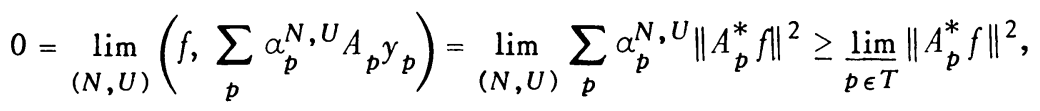

completing the proof.

Theorem 3. Let $X$ be a reflexive Banach space with $X$ and $X^{*}$ strictly convex. Let $\left(T_{n}\right)_{n \in S}$ be a net of contractions such that $x=\lim T_{n} x$ implies $x=T_{n} x$ eventually. Then the convergence set $M$ is the range of the linear contractive projection

$$
A=\lim _{N F \in Q_{N}} \lim _{p \rightarrow \infty} \lim _{k \rightarrow \infty} A_{p, k}^{F},
$$

where $Q_{N}$ is the set of finite subsets of the set of elements $p$ of $S, p \geq N$, directed under inclusion. $A_{p, k}^{F}$ is defined in Lemma 3.

Proof. $M=\bigcup_{N \in S} \bigcap_{n \geq N} N\left(I-T_{n}\right)$. By Corollary $1, M$ is the range of a linear contractive projection. Set $I-T=\left(I-T_{n}\right)_{n \in S^{\circ}}$ By Lemma $4, X=$ $M+\operatorname{cl} R(I-T)$. Given $\epsilon>0$, for $x$ in $M$ and $z$ in $c l R(I-T)$, take $y$ in $R(I-T),\|y-z\|<\epsilon / 3$, let $y=\lim _{N} \Sigma_{m} \alpha_{m}^{\left.N_{(} I-T_{n(m)}\right) y_{n(m)}}$, where $\left\|y_{n(m)}\right\|$ $\leq K$, take $N, \alpha_{m}^{N}$, such that $x \in N\left(I-T_{n}\right)$ for $n \geq N$ and $\left\|y-\Sigma_{m}^{N} \alpha_{m}^{N}\left(I-T_{n(m)}\right) y_{n(m)}\right\|$ $<\epsilon / 3$. Take $F_{N}$ the support of $\alpha_{m}^{N}, F=n\left(F_{N}\right)$, and set

$$
A_{p, k}^{F}=\frac{1}{p} \sum_{j=0}^{p-1}\left(\prod_{n \in F} T_{n}(k)\right)^{j}
$$

where $T_{n}(k)=(1 / k) \Sigma_{i=0}^{k-1} T_{n}^{i}$, and some order is chosen in the product. Choose $p$ and $q$ by Lemma 3 so that $\left\|A_{p, k}^{F} \sum \alpha_{m}^{N}\left(I-T_{n(m)}\right) y_{n(m)}\right\|<\epsilon / 3$. Then 


$$
\begin{aligned}
\left\|A_{p, k}^{F}(x+z)-x\right\| \leq & \left\|A_{p, k}^{F}(z-y)\right\|_{n}+\left\|A_{p, k}^{F}\left(y-\sum \alpha_{m}^{N}\left(I-T_{n(m)}\right) y_{n(m)}\right)\right\| \\
& +\left\|A_{p, k}^{F} \sum \alpha_{m}^{N}\left(I-T_{n(m)}\right) y_{n(m)}\right\| \\
\leq & \epsilon / 3+\epsilon / 3+\epsilon / 3
\end{aligned}
$$

proving the claim.

\section{REFERENCE}

1. S. J. Bernau, Theorems of Korovkin type for $L_{p}$ spaces, Pacific J. Math. (to appear).

DEPARTMENT OF MATHEMATICS, UNIVERSITY OF AUCKLAND, AUCKLAND, NEW ZEALAND 